\title{
12-month mortality and loss-to-program in antiretroviral-treated children: The leDEA pediatric West African Database to evaluate AIDS (pWADA), 2000-2008
}

Didier K Ekouevi ${ }^{1,2^{*}}$, Alain Azondekon ${ }^{3}$, Fatoumata Dicko ${ }^{4}$, Karen Malateste ${ }^{1}$, Pety Touré ${ }^{5,6}$, François T Eboua ${ }^{7}$, Kouakou Kouadio ${ }^{8}$, Lorna Renner ${ }^{9}$, Kevin Peterson ${ }^{10}$, François Dabis ${ }^{1}$, Haby Signaté Sy ${ }^{11}$, Valeriane Leroy ${ }^{1}$ and for the leDEA pediatric West Africa Working Group pWADA

\begin{abstract}
Background: The leDEA West Africa Pediatric Working Group (pWADA) was established in January 2007 to study the care and treatment of HIV-infected children in this region. We describe here the characteristics at antiretroviral treatment (ART) initiation and study the 12-month mortality and loss-to-program of HIV-infected children followed in ART programs in West Africa.

Methods: Standardized data from HIV-infected children followed-up in ART programs were included. Nine clinical centers from six countries contributed to the dataset (Benin, Côte d'Ivoire, Gambia, Ghana, Mali and Senegal). Inclusion criteria were the followings: age 0-15 years and initiated triple antiretroviral drug regimens. Baseline time was the date of ART initiation. WHO criteria was used to define severe immunosuppression based on CD4 count by age or CD4 percent $<15 \%$. We estimated the 12-month Kaplan-Meier probabilities of mortality and loss-toprogram (death or loss to follow-up $>6$ months) after ART initiation and factors associated with these two outcomes.

Results: Between June 2000 and December 2007, 2170 children were included. Characteristics at ART initiation were the following: median age of 5 years (Interquartile range (IQR: 2-9) and median CD4 percentage of 13\% (IQR: 7-19). The most frequent drug regimen consisted of two nucleoside reverse transcriptase inhibitors and one nonnucleoside reverse transcriptase inhibitors (62\%). During the first 12 months, 169 (7.8\%) children died and 461 (21.2\%) were lost-to-program. Overall, in HIV-infected children on ART, the 12-month probability of death was $8.3 \%$ (95\% Confidence Interval (Cl): 7.2-9.6\%), and of loss-to-program was 23.1\% (95\% Cl: 21.3-25.0\%). Both mortality and loss-to program were associated with advanced clinical stage, CD4 percentage $<15 \%$ at ART initiation and year (> 2005) of ART initiation.
\end{abstract}

Conclusion: Innovative and sustainable approaches are needed to better document causes of death and increase retention in HIV pediatric clinics in West Africa.

\footnotetext{
* Correspondence: didier.ekouevi@gmail.com

'INSERM, U897, \& Institut de Santé Publique, Epidémiologie et Développement (ISPED), Université Victor Segalen Bordeaux 2, Bordeaux, France

Full list of author information is available at the end of the article
} 


\section{Background}

Pediatric HIV infection is a worldwide public health challenge that disproportionately affects children in the poorest parts of the world. By December 2008, an estimated 33.4 million people were living with HIV, 2.1 million of whom were children under 15 years of age [1]. Furthermore, $16 \%$ of the 2.7 million new HIV infections in 2008 occurred in children [1]. At the end of 2009, 356,400 HIV-infected children younger than 15 years of age in low- and middle-income countries received antiretroviral therapy (ART) representing $28 \%$ of those in need of ART with a substantial variation within African region (12\% in Western and Central Africa and 32\% in Eastern and Southern Africa) [2].

Data from ongoing randomized clinical trials and observational datasets in Africa have demonstrated a clear mortality benefit from starting ART among children eligible for ART [3-9]. However, in sub-Saharan Africa, the daily management of HIV care for children remains a great challenge for the following reasons: first, diagnosing HIV infection early among children less than 18 months is difficult; second, human resources trained to deliver ART to children are lacking; third, pediatric antiretroviral formulations are limited; fourth, ART is complex in the context of multiple co-morbidities (tuberculosis, malaria, malnutrition, etc.); and lastly, low-income countries suffer from poor health care systems $[2,10,11]$. In spite of these challenges, data from various pediatric ART programs in Africa report good early clinical outcomes in children [3-5,8]. In adult cohorts, the poor retention, varied between $76-80 \%$, was reported at 12 months in HIV care among patients initiated ART [12-14]. Few reports on retention in pediatric care are available in subSaharan Africa.

In July 2006, the National Institute of Health launched the International epidemiological Database to Evaluate AIDS (IeDEA) initiative to better describe the trends of the epidemiology of HIV in the context of ART access in specific regions in the world http:// www.iedea-hiv.org In West Africa http://www.iedeawestafrica.org, the IeDEA Pediatric Working Group (pWADA) was established in January 2007 to study the care and treatment of HIV-infected children in this region of sub-Saharan Africa, where the ART coverage among children reported in 2009 was the lowest (12\%) [2].

In this context, we aimed to describe the characteristics of HIV-infected children at ART initiation and study the 12-month mortality and loss-to-program rates in the PWADA database.

\section{Methods}

The leDEA West Africa collaboration

By collecting and integrating data from multiple HIV/ AIDS cohorts, this initiative aims to address unique and evolving research questions in the field of HIV/AIDS care and treatment. In West Africa, this collaboration was initiated in July 2006 and currently involves 10 pediatric HIV/AIDS clinics spread over eight countries. The first merger, done in April 2008, included the data from nine pediatric clinical centers in Benin $(n=1)$, Côte d'Ivoire $(\mathrm{n}=4)$, Gambia $(\mathrm{n}=1)$, Ghana $(\mathrm{n}=1)$, Mali $(\mathrm{n}=1)$ and Senegal $(\mathrm{n}=1)$.

\section{Inclusion criteria}

In the IeDEA West Africa pediatric HIV center, clinical forms were available for recording HIV activities and an electronic database was available for data entry. We used a standardized data collection procedure to merge the HIV pediatric data [15]. All HIV-1-infected children (positive viral load test $<18$ months or positive serology $\geq 18$ months) aged $<16$ years with documented gender and initiated triple drugs antiretroviral therapy were included irrespective of the first-line ART regimens. The initiation of ART was based on the national or international guidelines $[11,16,17]$.

The following information was recorded in the database: age, gender, body mass index, date of ART initiation, pre-treatment $\mathrm{CD} 4$ percentage, cotrimoxazole, initiation and type of ART regimens. HIV-infected children are typically seen in clinics at least every three months, and their CD4 counts are measured twice a year to monitor their immunological response to ART. Routine viral load monitoring is not available in most sites.

\section{Ethical aspects}

The IeDEA West Africa collaboration was approved by the national ethics committees of each country. Individual informed signed consent was waived for this analysis.

\section{Outcomes}

The outcomes of this study were i) death, defined as any death recorded in the database during the first year of follow-up; ii) loss-to-follow-up, indicated when the interval between the last clinic visit registered in the database and the closing date of the database was $>6$ months; and iii) loss-to-program, defined as the combined outcome of death or loss-to-follow-up.

The baseline was the date of ART initiation. The explanatory variables included the following: gender, age at ART initiation, $2006 \mathrm{WHO}$ criteria for immunodeficiency 
according to CD4 cell count or CD4 percent and age [17], cotrimoxazole status at ART initiation, year of ART initiation and first-line antiretroviral regimen (NonNucleoside Reverse Transcriptase Inhibitor [NNRTI] or Protease Inhibitor [PI]). The clinical stage of the disease was defined as either less advanced (CDC stage A/B or WHO stage I/II/III/) or advanced (CDC stage $C$ or WHO stage IV).

\section{Statistical analysis}

Continuous variables were compared using the Wilcoxon rank-sum test, and comparisons between two categorical variables were performed using the Chisquare test and Fisher's exact test when appropriate. The Kaplan-Meier method was used to estimate the probability of death or loss to program with their 95\% Confidence Interval (CI). The logrank test was used for comparisons between groups. We used Weilbull regression models with random effects to estimate mortality and loss-to-program hazard ratios (HR) with their 95\% $\mathrm{CI}$, accounting for heterogeneity between the different programs or cohorts. Models included individual variables (age, sex, pre-ART CD4 count, type of initial regimen and clinical stage of disease, cotrimoxazole initiation and year of ART initiation). The multivariable analysis was performed by the backward selection procedure including all variables (explicative model). The dataset was closed for analysis after the data merger was completed in April 2008. Follow-up was censored at the date of death for children deceased or the date of the last visit for children who were lost to follow-up.
Children alive and in care in April 2008 were right-censored at the date of their last visit before this fixed date. All analyses were performed using intent-to-treat approach with SAS software version 9.1 (SAS Institute, Cary, NC, USA).

\section{Results}

\section{Description of sites}

The collaboration included nine pediatric clinical centers from six countries in West Africa (Table 1). Free access to all laboratory tests (CD4 count testing, liver function testing and total blood count) was available in three out of the nine clinical centers (Table 1). All of the children had free access to ART as well as cotrimoxazole prophylaxis expected at the Korbelu Teaching Hospital in Ghana. Four of the clinical centers are located within teaching hospitals. The biggest cohort was located in Bamako, with 673 ART-treated children, and the smallest site was in Gambia, with 23 children enrolled (Table 2).

\section{Characteristics of patients and antiretroviral regimens at ART initiation}

Between June 2000 and December 2007, 2,170 children were included. The baseline characteristics per center are summarized in table 2 . Overall, median age was 5 years (Interquartile range [IQR]: 2-9); median CD4 percentage was $13 \%$ (IQR: $7-19$ ); and $51.8 \%$ of these HIVinfected children had immunosuppression at ART initiation. All of the sites had similar baseline characteristics except for the MTCT-Plus clinic, which recruited

Table 1 Characteristics of the paediatric clinical sites in the leDEA West African collaboration (pWADA), $2000-2007$.

\begin{tabular}{|c|c|c|c|c|c|c|c|c|}
\hline Country & Site & $\begin{array}{l}\text { Electronic files } \\
\text { or medical } \\
\text { records }\end{array}$ & Location & $\begin{array}{l}\text { Sector } \\
\text { (public/ } \\
\text { private) }\end{array}$ & $\begin{array}{l}\text { Free } \\
\text { access } \\
\text { to ART }\end{array}$ & $\begin{array}{c}\text { Free access } \\
\text { to } \\
\text { cotrimoxazole }\end{array}$ & $\begin{array}{c}\text { Free } \\
\text { access to } \\
\text { lab exams* }\end{array}$ & $\begin{array}{l}\text { Availability of tracing } \\
\text { methods for loss to } \\
\text { follow-up** }\end{array}$ \\
\hline Benin & $\begin{array}{l}\text { Hôpital d'Instruction des } \\
\text { Armées, Cotonou (UPEIV) }\end{array}$ & Electronic & Urban & Private & Yes & Yes & Yes & Yes \\
\hline $\begin{array}{l}\text { Côte } \\
\text { d'Ivoire }\end{array}$ & $\begin{array}{l}\text { Centre de Prise en charge, de } \\
\text { Recherche et de Formation } \\
\text { (CEPREF), Abidjan }\end{array}$ & Electronic & Urban & Public & Yes & Yes & Yes & Yes \\
\hline $\begin{array}{l}\text { Côte } \\
\text { d'Ivoire }\end{array}$ & MTCT Plus Network, Abidjan & Electronic & Urban & Public & Yes & Yes & Yes & Yes \\
\hline $\begin{array}{l}\text { Côte } \\
\text { d'Ivoire }\end{array}$ & CHU de Yopougon, Abidjan & Electronic & Urban & Public & Yes & Yes & Yes & Yes \\
\hline $\begin{array}{l}\text { Côte } \\
\text { d'Ivoire }\end{array}$ & CIRBA & Electronic & Urban & $\begin{array}{l}\text { Semi- } \\
\text { public }\end{array}$ & Yes & Yes & Yes & No \\
\hline Gambia & Medical Research Council, Fajara & $\begin{array}{l}\text { Medical } \\
\text { records }\end{array}$ & Urban & Public & Yes & Yes & Yes & No \\
\hline Ghana & Korle Bu Hospital, Accra (KBTH) & $\begin{array}{l}\text { Medical } \\
\text { records }\end{array}$ & Urban & Public & No & No & Yes & No \\
\hline Senegal & $\begin{array}{c}\text { Hôpital d'Enfants Albert-Royer, } \\
\text { Dakar }\end{array}$ & $\begin{array}{l}\text { Medical } \\
\text { records }\end{array}$ & Urban & Public & Yes & Yes & Yes & Yes \\
\hline Mali & Hôpital Gabriel Touré, Bamako & Electronic & Urban & Public & Yes & Yes & Yes & No \\
\hline
\end{tabular}

* Free access to laboratory exams (CD4 count testing, total blood count, liver function testing)

**Tracing methods: used cell phone call or do home visit by social workers team when the patient missed one scheduled visit 
Table 2 Baseline characteristics at antiretroviral treatment (ART) initiation of 2170 children.

\begin{tabular}{|c|c|c|c|c|c|c|c|c|c|c|c|c|c|c|}
\hline \multirow[t]{2}{*}{ Site } & \multirow[t]{2}{*}{$\mathbf{N}$} & \multirow[t]{2}{*}{$\begin{array}{l}\text { Girl } \\
(\%)\end{array}$} & \multirow{2}{*}{$\begin{array}{c}\text { Median } \\
\text { Age } \\
\text { (years) } \\
\text { (IQR) }\end{array}$} & \multirow{2}{*}{$\begin{array}{l}\text { \%AIDS or } \\
\text { clinical } \\
\text { stage IV (\% } \\
\text { Missing) }\end{array}$} & \multicolumn{3}{|c|}{ Initial ART regimen (\%) } & \multirow{2}{*}{$\begin{array}{l}\text { Median } \\
\text { CD4 cell } \\
\text { count } \\
\text { (IQR) }\end{array}$} & \multirow{2}{*}{$\begin{array}{l}\text { Median } \\
\text { CD4 } \\
\text { percent } \\
\text { (IQR) }\end{array}$} & \multirow{2}{*}{$\begin{array}{l}\text { \%Immuno- } \\
\text { supression* } \\
\text { (\%Missing) }\end{array}$} & \multirow{2}{*}{$\begin{array}{c}\% C T X \\
\text { Status } \\
(\% \\
\text { Missing) }\end{array}$} & \multicolumn{3}{|c|}{$\begin{array}{l}\text { Year of ART } \\
\text { initiation }\end{array}$} \\
\hline & & & & & $\begin{array}{c}\text { 2NRTI } \\
+1 \text { NNRTI }\end{array}$ & $\begin{array}{l}2 \mathrm{NRTI} \\
+1 \mathrm{PI}\end{array}$ & Others & & & & & $\begin{array}{c}\leq \\
2004\end{array}$ & 2005 & $\begin{array}{c}\geq \\
2006\end{array}$ \\
\hline UPEIV & 71 & 46.5 & $4(2-7)$ & $24.3(1.4)$ & 0.0 & 100.0 & 0.0 & $\begin{array}{c}291 \\
(132-517)\end{array}$ & $8(5-12)$ & $89.9(2.8)$ & $\begin{array}{l}100.0 \\
(1.4)\end{array}$ & 50.7 & 19.7 & 29.6 \\
\hline CEPREF & 306 & 42.5 & $6(3-9)$ & $20.2(1.6)$ & 63.1 & 33.3 & 3.6 & $\begin{array}{c}356 \\
(106-728)\end{array}$ & $11(6-16)$ & $79.5(15.4)$ & $32.8(0.3)$ & 12.7 & 50.7 & 36.6 \\
\hline $\begin{array}{l}\text { MTCT } \\
\text { Plus }\end{array}$ & 74 & 52.7 & $2(1-4)$ & $10.3(21.6)$ & 35.1 & 64.9 & 0.0 & $\begin{array}{c}858 \\
(437-1285)\end{array}$ & $\begin{array}{c}18 \\
(14-24)\end{array}$ & $57.7(4.1)$ & $\begin{array}{l}89.7 \\
(21.6)\end{array}$ & 41.9 & 23.0 & 35.1 \\
\hline $\begin{array}{l}\text { CHU } \\
\text { Yopougon }\end{array}$ & 650 & 48.3 & $6(3-10)$ & $0.5(4.0)$ & 46.0 & 53.5 & 0.5 & $\begin{array}{c}445 \\
(201-738)\end{array}$ & $14(7-21)$ & $60.1(14.5)$ & $15.6(0.6)$ & 52.3 & 24.2 & 23.5 \\
\hline CIRBA & 141 & 47.5 & $4(2-7)$ & $53.3(5.1)$ & 66.7 & 31.9 & 1.4 & $\begin{array}{c}393 \\
(227-947)\end{array}$ & $14(6-20)$ & $72.0(46.8)$ & $\begin{array}{l}71.2 \\
(48.2)\end{array}$ & 13.5 & 19.9 & 66.7 \\
\hline FAJARA & 23 & 47.8 & $6(4-12)$ & - (100.0) & 82.6 & 13.0 & 4.3 & $\begin{array}{c}305 \\
(220-580)\end{array}$ & $15(9-19)$ & $50.0(4.3)$ & - (100.0) & 4.3 & 34.8 & 60.9 \\
\hline KBTH & 128 & 47.7 & $6(3-8)$ & $3.9(0.0)$ & 96.9 & 0.0 & 3.1 & $\begin{array}{c}332 \\
(77-684)\end{array}$ & $11(4-17)$ & $65.6(25.0)$ & $93.8(0.0)$ & 11.7 & 51.6 & 36.7 \\
\hline $\begin{array}{l}\text { Albert } \\
\text { Royer }\end{array}$ & 104 & 43.3 & $7(4-10)$ & $55.3(1.0)$ & 81.7 & 15.4 & 2.9 & $\begin{array}{c}340 \\
(31-610)\end{array}$ & $9(4-15)$ & $63.9(41.3)$ & $92.3(0.0)$ & 32.7 & 20.2 & 47.1 \\
\hline $\begin{array}{l}\text { Gabriel } \\
\text { Toure }\end{array}$ & 673 & 40.4 & $4(2-8)$ & $30.4(2.2)$ & 75.2 & 23.0 & 1.8 & $\begin{array}{c}335 \\
(115-602) \\
\end{array}$ & - & $55.8(16.3)$ & - (100.0) & 39.8 & 18.0 & 42.2 \\
\hline Total & 2170 & 44.8 & $5(2-9)$ & $19.1(13.2)$ & 62.0 & 36.3 & 1.7 & $\begin{array}{c}385 \\
(142-691)\end{array}$ & $\begin{array}{c}13 \\
(7-19)\end{array}$ & $63.4(18.3)$ & $\begin{array}{c}42.7 \\
(36.2)\end{array}$ & 36.0 & 27.1 & 36.9 \\
\hline
\end{tabular}

leDEA paediatric West Africa database (pWADA), 2000-2007.

IQR: Interquartile range, NRTI: Nucleoside reverse transcriptase inhibitors, NNRTI: Non nucleoside reverses transcriptase inhibitors, PI: protease inhibitors, KBTH: Korlebu Teaching Hospital, Accra, UPEIV: Hôpital d'Instruction des armées, Cotonou, CTX: Cotrimoxazole

* According to WHO definition [4], \$ at ART initiation

children at a younger median age of two years and a higher median CD4 percentage (18\%). Cotrimoxazole was initiated in $27.2 \%$ of children (range: $0.0 \%-98.6 \%$ ). The first-line treatment combinations were two nucleoside reverse transcriptase inhibitors (NRTI) and one non-nucleoside reverse transcriptase inhibitor (NNRTI) in $62.0 \%$ of the cases, followed by two NRTIs and one protease inhibitor (PI) in $36.3 \%$ of cases, and the other combinations were used in $1.7 \%$ of the cases. The most frequent PI used was Nelfinavir (95.7\%); the remaining cases were treated with Lopinavir boosted with Ritonavir.

\section{Mortality rate and loss-to-program}

Among these 2,170 HIV-infected children on ART, 169 $(7.8 \%)$ died during the follow-up period (1761 childyears) with a mortality rate, estimated to be 9.6 deaths per 100 child-years (95\% CI: 9.4-9.7). Overall, the 12month probability of death after ART initiation was 8.3\% (95\% CI: 7.2-9.6\%), with substantial differences across sites and age categories $(\mathrm{p}=0.001)$ (Table 3$)$. The 12 -month probability of death was $11.0 \%$ in children with severe immunosuppression (CD4 percentage $<15 \%$ ) at baseline, $2.7 \%$ in those with CD4 percentage $\geq$
15\% and $9.3 \%$ in those with unknown CD4 percentage $(\mathrm{p}<0.0001)$ (Figure 1).

The 12 month probability of lost-to follow-up was $16.0 \%$ (95\% CI: $14.4-17.8 \%$ ), and varied according to age group (Table 3).

A total of 461 children were lost-to-program (dead or last visit $>6$ months) with an incidence of 26.2 per 100 child-years, 95\% CI [25.9-26.4]. The 12-month probability of loss-to-program on ART were $23.1 \%$ (95\% CI: 21.3-25.0\%) overall and varied per age group $(\mathrm{p}=0.0005)($ Table 3$)$. This probability was $21.2 \%$ for a CD4 percentage $<15 \%, 10.8 \%$ for a $C D 4$ percentage $\geq 15 \%$ and $30.5 \%$ for missing CD4 percentage ( $\mathrm{c}$ 0.0001).

\section{Factors associated with death or loss-to-program}

A multivariable analysis was performed adjusting for gender, age, type of ART regimen, clinical stage, CD4 percentage of ART initiation, year of ART initiation, and cotrimoxazole status. The adjusted hazard ratio (aHR) of death was 2.1 (95\% CI: 1.1-4.1) for children with CD4 percentage < $15 \%$. It was 1.5 (95\% CI: 0.63.6) for those with a missing CD4 percentage (reference group was $C D 4 \geq 15 \%$ at ART initiation). The 
Table 3 Probability of death or loss to program in $\mathbf{2 1 7 0}$ children on antiretroviral therapy (ART), by age group at ART initiation.

\begin{tabular}{|c|c|c|c|c|c|c|c|c|c|c|c|c|}
\hline \multirow[b]{3}{*}{ Age at ART initiation } & \multicolumn{4}{|c|}{ Death } & \multicolumn{4}{|c|}{ Loss to follow-up } & \multicolumn{4}{|c|}{$\begin{array}{l}\text { Loss to program (death and } \\
\text { lost to follow-up) }\end{array}$} \\
\hline & \multicolumn{2}{|c|}{ Month-6 } & \multicolumn{2}{|c|}{ Month-12 } & \multicolumn{2}{|c|}{ Month-6 } & \multicolumn{2}{|c|}{ Month-12 } & \multicolumn{2}{|r|}{ Month-6 } & \multicolumn{2}{|c|}{ Month-12 } \\
\hline & $n$ & $\begin{array}{l}\text { Probability } \\
(95 \% \mathrm{Cl})\end{array}$ & $\mathrm{n}$ & $\begin{array}{l}\text { Probability } \\
(95 \% \mathrm{Cl})\end{array}$ & $\mathrm{n}$ & $\begin{array}{l}\text { Probability } \\
(95 \% \mathrm{Cl})\end{array}$ & $n$ & $\begin{array}{l}\text { Probability } \\
(95 \% \mathrm{Cl})\end{array}$ & $\mathrm{n}$ & $\begin{array}{l}\text { Probability } \\
(95 \% \mathrm{Cl})\end{array}$ & $n$ & $\begin{array}{c}\text { Probability } \\
(95 \% \mathrm{Cl})\end{array}$ \\
\hline$<12$ months & 36 & $\begin{array}{c}8.9 \\
(3.4 ; 22.2)\end{array}$ & 23 & $\begin{array}{c}11.7 \\
(5.0 ; 26.0)\end{array}$ & 36 & $\begin{array}{c}0.0 \\
(0.0 ; 0.0)\end{array}$ & 23 & $\begin{array}{c}20.2 \\
(10.2 ; 37.8)\end{array}$ & 36 & $\begin{array}{c}8.9 \\
(3.4 ; .22 .2)\end{array}$ & 23 & $\begin{array}{c}29.8 \\
(18.0 ; 46.6)\end{array}$ \\
\hline $1-3$ years & 398 & $\begin{array}{c}10.6 \\
(8.2 ; 13.7)\end{array}$ & 278 & $\begin{array}{c}12.7 \\
(10.0 ; 16.1)\end{array}$ & 398 & $\begin{array}{c}5.0 \\
(3.4 ; 7.3)\end{array}$ & 278 & $\begin{array}{c}19.4 \\
(15.8 ; 23.7)\end{array}$ & 398 & $\begin{array}{c}15.1 \\
(12.2 ; 18.5)\end{array}$ & 278 & $\begin{array}{c}29.7 \\
(25.7 ; 34.1)\end{array}$ \\
\hline 3-5 years & 341 & $\begin{array}{c}3.7 \\
(2.2 ; 6.2)\end{array}$ & 262 & $\begin{array}{c}5.2 \\
(3.4 ; 8.1)\end{array}$ & 341 & $\begin{array}{c}2.4 \\
(1.2 ; 4.6)\end{array}$ & 262 & $\begin{array}{c}15.9 \\
(12.3 ; 20.3)\end{array}$ & 341 & $\begin{array}{c}6.0 \\
(4.0 ; 8.9)\end{array}$ & 262 & $\begin{array}{c}20.3 \\
(16.5 ; 24.9)\end{array}$ \\
\hline $5-10$ years & 629 & $\begin{array}{c}5.6 \\
(4.2 ; 7.5)\end{array}$ & 516 & $\begin{array}{c}7.1 \\
(5.4 ; 9.2)\end{array}$ & 629 & $\begin{array}{c}4.2 \\
(3.0 ; 6.0)\end{array}$ & 516 & $\begin{array}{c}13.8 \\
(11.4 ; 16.8)\end{array}$ & 629 & $\begin{array}{c}9.6 \\
(7.7 ; 12.0)\end{array}$ & 516 & $\begin{array}{c}19.9 \\
(17.1 ; 23.1)\end{array}$ \\
\hline$\geq 10$ years & 376 & $\begin{array}{c}6.2 \\
(4.3 ; 8.9)\end{array}$ & 294 & $\begin{array}{c}8.1 \\
(5.9 ; 11.2)\end{array}$ & 376 & $\begin{array}{c}4.3 \\
(2.7 ; 6.7)\end{array}$ & 294 & $\begin{array}{c}15.8 \\
(12.5 ; 19.9)\end{array}$ & 376 & $\begin{array}{c}10.2 \\
(7.7 ; 13.5)\end{array}$ & 294 & $\begin{array}{c}22.7 \\
(18.9 ; 27.0)\end{array}$ \\
\hline
\end{tabular}

leDEA pediatric West Africa database (pWADA), 2000-2007.

$\mathrm{n}$ : number of children at risk

aHR was 2.5 (95\% CI: 1.6-3.9) for children at an advanced clinical stage at ART initiation. For children who initiated ART in 2005, the aHR was 1.7 (95\% CI: 1.0-2.9) compared to those who initiated ART before 2005. The aHR was 2.8 (95\% CI: 1.7-4.7) for children who initiated ART in 2006 or after. The remaining variables such as gender, age, first-line ART regimen and cotrimoxazole status were not independently associated with death (Table 4).

Similar factors were associated with loss-to-program (Table 5): advanced clinical stage $(\mathrm{aHR}=2.3,95 \% \mathrm{CI}$ $(1.7-3.0))$; CD 4 percentage $<15 \%(\mathrm{aHR}=1.9,95 \% \mathrm{CI}$ (1.3-2.9)); and year of ART initiation. The aHR for children who initiated ART in 2005 was 1.7 (95\% CI: 1.3-
(A)

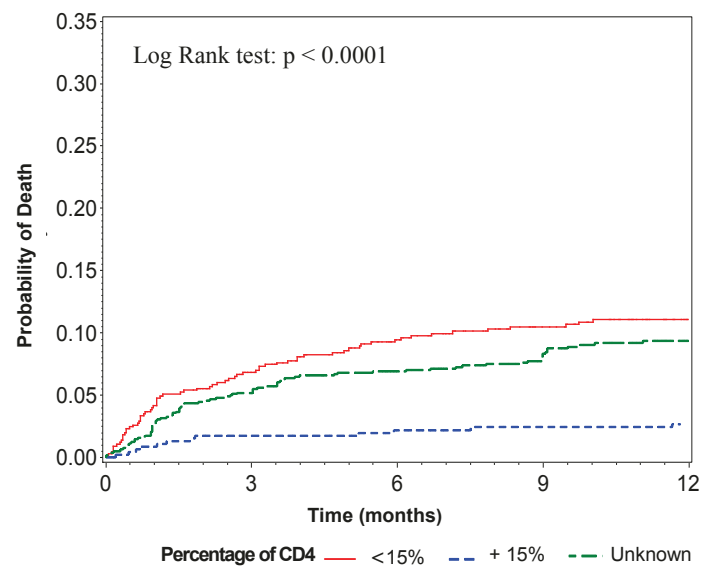

(B)

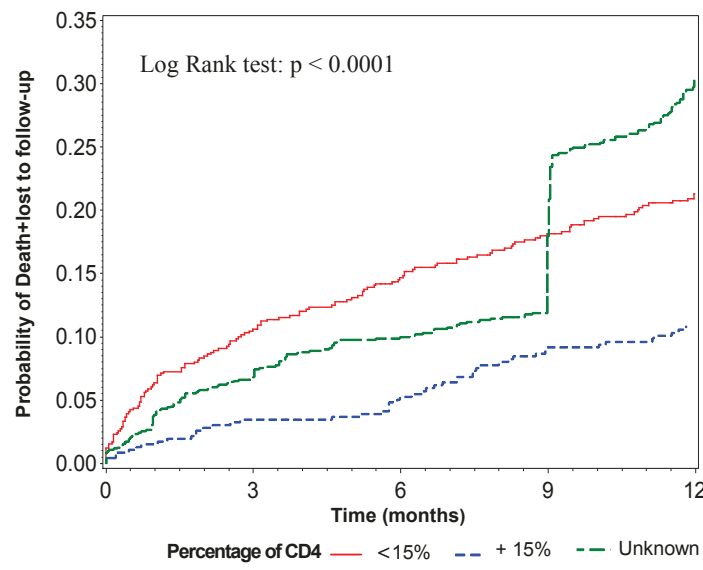

\begin{tabular}{|c|c|c|c|c|c|c|c|c|}
\hline \multirow{2}{*}{$\begin{array}{l}\text { Percentage of } \\
\text { CD4 at baseline }\end{array}$} & \multicolumn{2}{|c|}{ Month-6 } & \multicolumn{2}{|r|}{ Month-12 } & \multicolumn{2}{|c|}{ Month-6 } & \multicolumn{2}{|c|}{ Month-12 } \\
\hline & $\begin{array}{c}\text { Children } \\
\text { at risk }\end{array}$ & $\begin{array}{c}\text { Probability } \\
(95 \% \mathrm{CI})\end{array}$ & $\begin{array}{c}\text { Children } \\
\text { at risk }\end{array}$ & $\begin{array}{c}\text { Probability } \\
(95 \% \mathrm{CI})\end{array}$ & $\begin{array}{c}\text { Children } \\
\text { at risk }\end{array}$ & $\begin{array}{c}\text { Probability } \\
(95 \% \mathrm{CI})\end{array}$ & $\begin{array}{c}\text { Children } \\
\text { at risk }\end{array}$ & $\begin{array}{c}\text { Probability } \\
(95 \% \mathrm{CI})\end{array}$ \\
\hline$<15 \%$ & 531 & $9.4(7.4 ; .12 .0)$ & 439 & $11.0(8.8 ; 13.8)$ & 531 & $14.6(12.2 ; 17.6)$ & 439 & $21.2(18.2 ; 24.6)$ \\
\hline$\geq 15 \%$ & 417 & $2.2(1.2 ; 4.0)$ & 372 & $2.7(1.5 ; 4.7)$ & 417 & $5.2(3.5 ; 7.7)$ & 372 & $10.8(8.2 ; 14.1)$ \\
\hline Unknown & 832 & $6.9(5.5 ; 8.7)$ & 562 & $9.3(7.6 ; 11.4)$ & 832 & $10.0(8.3 ; 12.0)$ & 562 & $30.5(27.5 ; 33.6)$ \\
\hline
\end{tabular}

Baseline: at ART initiation

Figure 1 Kaplan-Meier probability of death (A) or loss to program (death or loss to follow-up) (B) in 2170 children on antiretroviral therapy (ART), by CD4 percentage at ART initiation. leDEA pediatric West Africa database (pWADA), 2000-2007. 
Table 4 Weibull survival model with random effects in 2131 children on antiretroviral therapy (ART).

\begin{tabular}{|c|c|c|c|c|c|c|}
\hline & \multicolumn{3}{|c|}{ Univariate analysis } & \multicolumn{3}{|c|}{ Multivariate analysis } \\
\hline & $\mathrm{HR}^{*}$ & $95 \% \mathrm{Cl}$ & $P$ & aHR & $95 \% \mathrm{Cl}$ & $P$ \\
\hline \multicolumn{7}{|l|}{ Sex } \\
\hline Boys & 1 & - & - & 1 & - & - \\
\hline Girls & 1.1 & $0.7-1.5$ & 0.72 & 1.0 & $0.7-1.4$ & 0.88 \\
\hline \multicolumn{7}{|l|}{ Age at ART initiation } \\
\hline 10 years and plus & 1 & - & - & 1 & - & - \\
\hline [5-10] years & 0.8 & $0.5-1.4$ & 0.49 & 0.9 & $0.5-1.5$ & 0.70 \\
\hline$[3-5]$ years & 0.6 & $0.3-1.2$ & 0.15 & 0.9 & $0.5-1.7$ & 0.68 \\
\hline$[1-3]$ years & 1.5 & $0.9-2.6$ & 0.12 & 1.2 & $0.7-2.1$ & 0.40 \\
\hline$<12$ months & 1.2 & $0.4-4.3$ & 0.69 & 1.1 & $0.3-3.8$ & 0.79 \\
\hline \multicolumn{7}{|c|}{ ART regimen at baseline } \\
\hline $2 \mathrm{NRTI}+1 \mathrm{NNRTI}$ & 1 & - & - & 1 & & \\
\hline $2 \mathrm{NRTI}+1 \mathrm{Pl}$ & 0.7 & $0.4-1.0$ & 0.06 & 0.9 & $0.6-1.5$ & 0.76 \\
\hline Other & 0.2 & $0.02-2.6$ & 0.21 & 1.0 & $0.2-5.0$ & 0.98 \\
\hline \multicolumn{7}{|c|}{ Clinical stage at ART initiation } \\
\hline A, B or I, II, III & 1 & - & - & 1 & - & - \\
\hline AIDS or IV & 6.0 & $3.9-9.2$ & $<0.01$ & 2.5 & $1.6-3.9$ & $<0.01$ \\
\hline Unknown & 0.9 & $0.4-2.0$ & 0.90 & 1.4 & $0.7-2.9$ & 0.29 \\
\hline \multicolumn{7}{|c|}{$\%$ CD4 at ART initiation } \\
\hline$\geq 15 \%$ & 1 & - & - & 1 & - & - \\
\hline$<15 \%$ & 2.1 & $1.1-3.9$ & 0.02 & 2.1 & $1.1-4.1$ & 0.03 \\
\hline Missing & 1.5 & $0.7-3.3$ & 0.22 & 1.5 & $0.6-3.6$ & 0.27 \\
\hline \multicolumn{7}{|l|}{ Year of ART initiation } \\
\hline$\leq 2004$ & 1 & - & - & 1 & - & - \\
\hline 2005 & 1.8 & $1.0-3.0$ & 0.03 & 1.7 & $1.0-2.9$ & 0.04 \\
\hline$\geq 2006$ & 2.6 & $1.7-4.2$ & $<0.01$ & 2.8 & $1.7-4.7$ & $<0.01$ \\
\hline \multicolumn{7}{|c|}{ Initiation of cotrimoxazole at ART initiation } \\
\hline No & 1 & - & - & 1 & - & - \\
\hline Yes & 1.3 & $0.7-2.5$ & 0.39 & 0.9 & $0.5-1.7$ & 0.67 \\
\hline Missing & 0.6 & $0.1-3.1$ & 0.50 & 0.4 & $0.1-2.3$ & 0.30 \\
\hline
\end{tabular}

leDEA paediatric West Africa database (pWADA), 2000-2007. Mortality analysis.

${ }^{*} \mathrm{HR}=$ Hazard Ratio, ${ }^{* *} 95 \% \mathrm{Cl}=95 \%$ Confidence Interval, aHR = adjusted Hazard Ratio

Heterogeneity between cohort, $p=0.25$

2.4), and the aHR for those who initiated ART in 2006 and after was 3.0 (95\% CI: 2.2-4.0) compared to those who initiated ART before 2005

\section{Discussion}

The IeDEA West Africa collaboration provides an opportunity to collect, analyze and compare access to ART and its field outcomes in this part of the world. After one year on ART, the overall mortality in children was $8.3 \%$ and the retention in HIV care programs was $78.8 \%$. The same factors were independently associated with both death and retention in HIV care: an advanced HIV disease, a severe immunosuppression at ART initiation and year of ART initiation.

Similar mortality rates was reported in other studies conducted with HIV-infected children in Africa and, ranging from $6.3 \%$ to $11.5 \%[3,5,7,8,18]$. The mortality rate reported in the studied cohorts is likely under-estimated because there was a high rate of loss-to-follow-up in the HIV cohort and lost to follow-up children were severely immune-suppressed, a marker of a higher risk of death. In general, mortality is under-estimated in ART African programs because of the misclassification of LTFU $[12,19,20]$. An investigation conducted in adult patients LTFU in Malawi found that $50 \%$ of them had died, $23 \%$ were alive and $27 \%$ untraceable [21]. We were not able to classify patients LTFU as their status was not routinely ascertained in the participating centers.

All the participating cohorts reported a late access to ART, except the Abidjan MTCT-Plus group, in which the linkage between PMTCT and HIV postnatal care has been strengthened by early HIV pediatric diagnosis. In the MTCT-Plus group, the median age at ART initiation was 2 years [22]. Indeed, in the current study, only 
Table 5 Weibull survival model with random effects in 2131 children on antiretroviral therapy (ART).

\begin{tabular}{|c|c|c|c|c|c|c|}
\hline & \multicolumn{3}{|c|}{ Univariate analysis } & \multicolumn{3}{|c|}{ Multivariate analysis } \\
\hline & $\mathrm{HR}^{*}$ & $\mathrm{Cl}(95 \%)^{* *}$ & $P$ & aHR & $\mathrm{Cl}(95 \%)^{* *}$ & $P$ \\
\hline \multicolumn{7}{|l|}{ Sex } \\
\hline Boys & 1 & - & - & 1 & - & - \\
\hline Girls & 1.0 & $0.8-1.3$ & 0.72 & 1.0 & $0.8-1.2$ & 0.78 \\
\hline \multicolumn{7}{|l|}{ Age at ART initiation } \\
\hline 10 years and plus & 1 & - & - & 1 & - & - \\
\hline [5-10] years & 0.9 & $0.6-1.2$ & 0.32 & 0.9 & $0.7-1.3$ & 0.56 \\
\hline$[3-5]$ years & 0.8 & $0.5-1.2$ & 0.22 & 0.9 & $0.6-1.3$ & 0.52 \\
\hline$[1-3]$ years & 1.3 & $0.9-1.8$ & 0.10 & 1.1 & $0.8-1.6$ & 0.34 \\
\hline$<12$ months & 1.4 & $0.6-2.9$ & 0.36 & 1.1 & $0.5-2.4$ & 0.66 \\
\hline \multicolumn{7}{|c|}{ ART regimen at baseline } \\
\hline $2 \mathrm{NRTI+1} \mathrm{NNRTI}$ & 1 & - & - & 1 & & \\
\hline $2 \mathrm{NRTI+1}$ IP & 0.7 & $0.5-0.9$ & 0.01 & 0.9 & $0.7-1.2$ & 0.62 \\
\hline Other & 0.6 & $0.2-1.5$ & 0.24 & 1.0 & $0.4-2.5$ & 0.98 \\
\hline \multicolumn{7}{|c|}{ Clinical stage at ART initiation } \\
\hline A, B or I, II, III & 1 & - & - & 1 & - & - \\
\hline AIDS or IV & 2.6 & $2.0-3.5$ & $<0.01$ & 2.3 & $1.7-3.0$ & $<0.01$ \\
\hline Unknown & 1.3 & $0.8-2.1$ & 0.21 & 1.4 & $0.9-2.3$ & 0.15 \\
\hline \multicolumn{7}{|l|}{$\%$ CD4 at ART initiation } \\
\hline$\geq 15 \%$ & 1 & - & - & 1 & - & - \\
\hline$<15 \%$ & 2.1 & 1.4.3.2 & $<0.01$ & 1.9 & $1.3-2.9$ & $<0.01$ \\
\hline Missing & 1.5 & $0.9-2.5$ & 0.07 & 1.7 & $1.1-2.8$ & 0.03 \\
\hline \multicolumn{7}{|l|}{ Year of ART initiation } \\
\hline$\leq 2004$ & 1 & - & - & 1 & - & - \\
\hline 2005 & 2.0 & $1.5-2.7$ & $<0.01$ & 1.7 & $1.3-2.4$ & $<0.01$ \\
\hline$\geq 2006$ & 3.5 & $2.6-4.6$ & $<0.01$ & 3.0 & $2.2-4.0$ & $<0.01$ \\
\hline \multicolumn{7}{|c|}{ Cotrimoxazole status at ART initiation } \\
\hline No & 1 & - & - & 1 & - & - \\
\hline Yes & 1.2 & $0.7-1.8$ & 0.43 & 0.9 & $0.6-1.4$ & 0.63 \\
\hline Missing & 0.6 & $0.2-1.8$ & 0.32 & 0.4 & $0.1-1.5$ & 0.16 \\
\hline
\end{tabular}

leDEA paediatric West Africa database (pWADA), 2000-2007. Loss-to-program analysis.

${ }^{*} \mathrm{HR}=$ Hazard Ratio, ${ }^{*} 95 \% \mathrm{Cl}=95 \%$ Confidence Interval, aHR = adjusted Hazard Ratio

Heterogeneity between cohort, $\mathrm{p}=0.14$

$2 \%$ of children initiated ART before their first year age. This observation is consistent with data reported by MSF programs, which reported that only $8.5 \%$ of children were less than 12 months at ART initiation [8]. Our findings underline important concerns in the timely identification of HIV-infected children in urban areas of West Africa. The linkage between the PMTCT program and pediatric care is crucial and should be reinforced wherever possible to identify HIV-infected infants earlier [22]. Substantial challenges in pediatric care in lowincome countries include identifying HIV-infected children earlier and initiating ART treatment as quickly as possible. The access to an early infant diagnostic for HIV by PCR remains largely uncommon [23,24]. Despite the recent availability of dried blood spots testing, including in rural areas [23-25], the delay between HIV testing and availability of the results remains a concern and requires particular organization. In 2009, only $15 \%$ of children born to HIV-positive mothers received an HIV test within the two first two months of life [2].

In the current study, age was not associated with death as reported in the KIDS-ART-LINC Collaboration [3] and also with loss-to-program. We unquestionably enrolled in this observational data the HIV-1 infected long-term non-progressors who survive until 5 years as most of HIV transmission among infants were motherto-child transmission. Indeed, the mortality in verticallyinfected children before the introduction of ART in the first year of life was about $40 \%$ and less than $30 \%$ in children infected in postnatal period [26].

We reported that $51.8 \%$ of children exhibited severe immunosuppression at ART initiation, which was associated with both death and loss-to-program. This association was previously reported for death but not for 
loss to follow-up [3,27]. The long delay before the diagnostic of pediatric HIV infection in children could also explain the severe immunosuppression observed among HIV-infected children who initiated ART.

HIV-infected children who initiated ART in 2005 or after presented a higher risk of death or loss-to-program compared to children who initiated ART before 2005. Possible explanations for this finding are as follows. First, the children who initiated ART after 2005 were at a more advanced clinical stage and presented more severe immunosuppression than the children who initiated before 2005 (data not shown). Second, there was a substantial increase in the number of HIVinfected children who were followed up in these centers with an increased workload, which may have impacted the standard of care. Indeed, we noted that the number the children who initiated ART between 2005 and 2008 roughly doubled in this period. Third, after 2005, there was an extension of new pediatric clinical centers with the new initiatives and support of US partners. It suggests that existing services are increasingly challenged to cope with the increased load of HIV-infected children due to the rapid scale up of ART programs in West African countries.

In the current study, retention of HIV pediatric care programs was $78.8 \%$ in West Africa at 12 months of follow-up. Retention was $85 \%$ at 12 months and $75 \%$ at 36 months in the pediatric MSF programs [8]. Retention in HIV care is now one of the most important challenges faced by heath care workers and HIV implementing partners as the coverage of HIV care and treatment have improved for both adults and children in lowincome countries. Similar findings were reported in adult patients, with a low rate (76\%) of retention in HIV care at 12 months in the IeDEA West Africa [12]. Also, a systematic review of adult patients in low-income countries reported that the retention in HIV care at 36 months was $64.6 \%$ [13]. Innovative and sustainable approaches to improve retention in HIV pediatric care are needed. For the adult's HIV-infected patients, then keys recommendations was proposed based on the Malawi's' experience [28]. We believe that some of these recommendations could be applied for the pediatric HIV care.

We acknowledge that the present study is not representative of all children on ART in West Africa as most of the data was gathered from urban sites, in which the standard of care may be higher than in rural areas. However, this study allows a comparison of the management of HIV care at the patient level between the programs in six countries in West Africa. In addition, we hypothesize that the operational problems identified here will be worse in less structured clinical care.
One limitation of this study, and generally in large collaborative studies, is the lack of data collected on factors related to access to health care, such as adherence to ART, or social variables, such as orphan status, to better explain mortality in HIV-infected children. These key variables are not routinely collected in the clinical forms used in the different HIV centers. In addition, the analysis did not include nutritional status (weight-forage, height-for-age) as the data available are not exploitable due to important missing data. Also, data on cotrimoxazole initiation was under-reported, explaining the low rate of children who initiated cotrimoxazole in the collaboration. Another limitation is the quality of data in the HIV cohort in West Africa, specifically in regards to missing data of key variables, such as percentage of CD4 count at baseline (47\%). The quality of data collected as well as its validity remains challenging in low incomes countries. However, this merger of data presented a clear picture of the quality of data and provided an example of the challenges of gathering reliable data in the field reality of the HIV pediatric cohorts in West Africa. After the assessment of available data, procedures were put into place in the collaboration to improve the quality of data in the participating centers.

There have been dramatic advances in the daily management of HIV disease in children in West Africa, with overall good clinical outcomes but low retention rates. The IeDEA West Africa collaboration offers the opportunity to increase the quality of data, which remains a concern in sub-Saharan African cohort, as well as to better document clinical outcomes. Comparisons with other IeDEA regions of the world could improve the understanding of the clinical outcomes reported.

\section{Conclusions}

It is urgent to find innovative approaches to early screening of HIV-infected children from six weeks of age onwards to implement large scale and durable pediatric ART programs. It is also important to better document causes of death and loss to program and to propose sustainable approaches to increase retention in HIV pediatric programs in West Africa.

\footnotetext{
Acknowledgements

The International epidemiological Database to Evaluate AIDS in West Africa (leDEA West Africa) is supported by the National Institute of Allergy and Infectious Diseases (NIAID) of the U.S. National Institutes of Health (NIH) as part of the International Epidemiologic Databases to Evaluate AIDS (leDEA) (grant no. 5U01Al069919-01 to 05). The content of this publication is solely the responsibility of the authors and does not necessarily represent the official views of any of the institutions mentioned above. The leDEA West Africa Working Group is organized as follows: - Primary Investigators: Pr François Dabis* (INSERM U897, ISPED, Bordeaux, France), Pr Emmanuel Bissagnene* (SMIT, CHU de Treichville, Abidjan, Côte d'Ivoire)
} 
- Clinical Investigators by country and alphabetical order (* Member of the leDEA West Africa Technical Committee): Jocelyn Akakpo, Alain Azondékon, Jules Bashi, Sagbo Gratien, Sikiratou Koumakpaï, Marcel D. Zannou* (Benin); Ye Diarra, Eric-Arnaud Diendere, Joseph Drabo*, Fla Koueta (Burkina Faso); Edmond Aka-Addi, Clarisse Amani-Bosse, Franck-Olivier Ba-Gomis, François Eboua-Tanoh, Serge-Paul Eholie*, Calixte Guehi, Kouakou Kouadio, SergeOlivier Koulé, Eugène Messou, Albert Minga, Aristophane Tanon, Marguerite Timité-Konan, Pety Touré, (Côte d'Ivoire); Kevin Peterson* (Gambia); Bamenla Goka, Lorna Renner* (Ghana); Hadizatou Coulibaly, Fatoumata Dicko, Moussa Maiga*, Daouda Minta, Mariam Sylla, Hamar Alassane Traoré (Mali); Man Charurat* (Nigeria); Bernard Diop, Fatou Ly Ndiaye, Papa Salif Sow, Haby Signaté Sy*, Judicaël Tine (Senegal)

- Epidemiology and Statistical Unit (INSERM U897, ISPED, Université Victor Segalen, Bordeaux, France): Eric Balestre, Didier K. Ekouévi*, Antoine Jaquet*, Valériane Leroy*, Charlotte Lewden*, Karen Malateste, Annie Sasco, Rodolphe Thiebaut

- Data Management Unit (PACCl, CHU Treichville, Abidjan, Côte d'Ivoire): Gérard Allou, Jean Claude Azani, Patrick Coffie

- Adult clinical centers by city and country:

Abidjan, Côte d'Ivoire: Médecine Interne et Tropicale (SMIT), CHU de Treichville, Unité de Soins Ambulatoires et de Conseil (USAC), Centre Médical de Suivi de Donneurs de Sang/CNTS/PRIMO-Cl, ACONDA-MTCT-Plus, ACONDA-CePReF, Centre Intégré de Recherche Bioclinique d'Abidjan (CIRBA) Abuja, Nigeria: University of Abuja Teaching Hospital (UATH) Bamako, Mali: Service d'Hépato-Gastro-Entérologie, Hôpital Gabriel Touré, Centre de Prise en Charge des Personnes vivant avec le VIH, Hôpital du Point $G$

Dakar, Sénégal: Service des Maladies Infectieuses, CHU de FANN/ISAARV Banjul, Gambia: Fajara Cohort

Benin City, Nigeria: University of Benin Teaching Hospital (UBTH) Cotonou, Benin: Service de Médecine Interne, CNHU Hubert Maga Ouagadougou, Burkina-Faso: Service de Médecine Interne, CHU Yalgado - Pediatric clinical centers by city and country:

Abidjan, Côte d'Ivoire: ACONDA-CEPREF, ACONDA-MTCT-Plus, CHU de Yopougon, Centre Intégré de Recherche Bioclinique d'Abidjan (CIRBA) Accra, Ghana: Korle Bu Teaching Hospital

Bamako, Mali: Hôpital Gabriel Touré

Cotonou, Benin: Centre National Hospitalo-Universitaire Hubert Maga, Hôpital d'Instruction des Armées.

Dakar, Senegal: Hôpital d'Enfants Albert-Royer

Fajara, Gambia: Medical Research Council

Ouagadougou, Burkina-Faso: Centre Hospitalier Charles de Gaulle.

- Administration: Alexandra Doring and Elodie Rabourdin (ISPED), Hughes

Djétouan, Bertin Kouadio and Adrienne Kouakou (PACCI)

\section{Author details}

'INSERM, U897, \& Institut de Santé Publique, Epidémiologie et Développement (ISPED), Université Victor Segalen Bordeaux 2, Bordeaux, France. 2Programme PAC-Cl, CHU de Treichville, Abidjan, Côte d'Ivoire. ${ }^{3}$ Hôpital d'Instruction des Armées, Cotonou, Benin. ${ }^{4}$ Hôpital Gabriel Touré, Service de Pédiatrie Bamako, Mali. ${ }^{5}$ MTCT-Plus initiative, ACONDA, Abidjan, Côte d'Ivoire. ${ }^{6} \mathrm{CePReF}$, ACONDA, Abidjan Côte d'Ivoire. ${ }^{7} \mathrm{CHU}$ de Yopougon, Service de Pédiatrie, Abidjan, Côte d'Ivoire. ${ }^{8}$ Centre Intégré de Recherche Bioclinique d'Abidjan (CIRBA), Abidjan, Côte d'Ivoire. ${ }^{9}$ Korlebu Hospital, Pediatric ward, Accra, Ghana. ${ }^{10}$ Medical Research Council, Fajara Cohort, Banjul, Gambia. ${ }^{11}$ Hôpital d'Enfants Albert-Royer, Dakar, Senegal.

\section{Authors' contributions}

DKE, AA, KM and VL conceived of the study, and participated in its design and coordination. KM and VL performed the statistical analysis. HS, PT, KK, FET participated at the design of the study and its coordination. DKE, FD, LR, $\mathrm{KP}$ and VL drafted the manuscript. All authors read and approved the final manuscript

\section{Competing interests}

The authors declare that they have no competing interests.
Received: 26 October 2010 Accepted: 30 June 2011

Published: 30 June 2011

\section{References}

1. UNAIDS: AIDS epidemic update 2009.[http://data.unaids.org/pub/Report/ 2009/JC1700_Epi_Update_2009_en.pdf], Accessed date 10 May 2011.

2. WHO/UNAIDS/UNICEF: Towards universal access. Scaling up priority HIV/ AIDS interventions in the health sector. Progress Report, 2010.[http:// www.who.int/hiv/pub/2010progressreport/report/en/index.html], Accessed date 10 May 2011.

3. KIDS-ART-LINC Collaboration: Low risk of death, but substantial program attrition, in pediatric HIV treatment cohorts in Sub-Saharan Africa. $J$ Acquir Immune Defic Syndr 2008, 49(5):523-31.

4. Anaky MF, Duvignac J, Wemin L, Kouakoussui A, Karcher S, Touré S, Seyler C, Fassinou P, Dabis F, N'Dri-Yoman T, Anglaret X, Leroy V: Scaling up antiretroviral therapy for HIV-infected children in Cote d'Ivoire: determinants of survival and loss to programme. Bull World Health Organ 2010, 88(7):490-9.

5. Bolton-Moore C, Mubiana-Mbewe M, Cantrell RA, Chintu N, Stringer EM, Chi BH, Sinkala M, Kankasa C, Wilson CM, Wilfert CM, Mwango A, Levy J, Abrams EJ, Bulterys M, Stringer JS: Clinical outcomes and CD4 cell response in children receiving antiretroviral therapy at primary health care facilities in Zambia. JAMA 2007, 298(16):1888-99.

6. Davies MA, Keiser O, Technau K, Eley B, Rabie H, van Cutsem G, Giddy J, Wood R, Boulle A, Egger M, Moultrie H, International Epidemiologic Databases to Evaluate AIDS Southern Africa (leDEA-SA) Collaboration: Outcomes of the South African National Antiretroviral Treatment Programme for children: the leDEA Southern Africa collaboration. S Afr Med J 2009, 99(10):730-7.

7. Fassinou P, Elenga N, Rouet F, Laguide R, Kouakoussui KA, Timite M, Blanche S, Msellati P: Highly active antiretroviral therapies among HIV-1infected children in Abidjan, Cote d'Ivoire. AIDS 2004, 18(14):1905-13.

8. Sauvageot D, Schaefer M, Olson D, Pujades-Rodriguez M, O'Brien DP: Antiretroviral therapy outcomes in resource-limited settings for HIVinfected children $<5$ years of age. Pediatrics 125(5):e1039-47.

9. Violari A, Cotton MF, Gibb DM, Babiker AG, Steyn J, Madhi SA, JeanPhilippe P, McIntyre JA, CHER Study Team: Early antiretroviral therapy and mortality among HIV-infected infants. N Engl J Med 2008, 359(21):2233-44.

10. World Health Organisation: WHO HIV prevention and treatment guidelines. Guidelines for co-trimoxazole prophylaxis for HIV-related infections in children, adults and adolescents in resource-limited settings: recommendations for a public health approach. Geneva: WHO; 2006 [http://www.who.int/hiv/pub/guidelines/ctx/en/index.html], Accessed date 10 May 2011

11. WHO: Paediatric HIV/antiretroviral therapy care guideline review. Geneva, Switzerland:[http://www.who.int/hiv/pub/paediatric/art_meeting_april2008/ en/index.html], WHO technical reference group meeting. 10-11 April 2008, Accessed date 10 May 2011.

12. Ekouevi DK, Balestre E, Ba-Gomis FO, Eholie SP, Maiga M, Amani-Bosse C, Minga A, Messou E, Sow PS, Lewden C, Traoré HA, Bissagnene E, Dabis F, IeDEA West Africa Collaboration: Low retention of HIV-infected patients on antiretroviral therapy in 11 clinical centres in West Africa. Trop Med Int Health 2011, 15(Suppl 1):34-42.

13. Fox MP, Rosen S: Patient retention in antiretroviral therapy programs up to three years on treatment in sub-Saharan Africa, 2007-2009: systematic review. Trop Med Int Health 2011, 15(Suppl 1):1-15.

14. Rosen S, Fox MP, Gill CJ: Patient retention in antiretroviral therapy programs in sub-Saharan Africa: a systematic review. PLoS Med 2007, 4(10):e298.

15. Kjaer J, Ledergerber B: HIV cohort collaborations: proposal for harmonization of data exchange. Antivir Ther 2004, 9(4):631-3.

16. WHO: Care, treatment, and support for women living with HIV/AIDS and their children in resource-constrained settings. Antiretroviral drugs for treatment in pregnant women and for prevention of HIV infection in infants and young children 2004 [http:/www.who.int/hiv/pub/mtct/guidelines/en/], Revision, Accessed date 10 May 2011.

17. WHO: Antiretroviral therapy of HIV infection in infants and children in resource-limited settings: towards universal access. 2006 [http://www. who.int/hiv/pub/guidelines/art/en/index.html], Accessed date 10 May 2011.

18. Rouet F, Fassinou P, Inwoley A, Anaky MF, Kouakoussui A, Rouzioux C, Blanche S, Msellati P: Programme Enfants Yopougon. Long-term survival 
and immuno-virological response of African HIV-1-infected children to highly active antiretroviral therapy regimens. AIDS 2006, 20(18):2315-9, ANRS 1244/1278

19. Brinkhof MW, Dabis F, Myer L, Bangsberg DR, Boulle A, Nash D, Schechter M, Laurent C, Keiser O, May M, Sprinz E, Egger M, Anglaret X, ART-LINC, leDEA: Early loss of HIV-infected patients on potent antiretroviral therapy programmes in lower-income countries. Bull World Health Organ 2008, 86(7):559-67.

20. Brinkhof MW, Pujades-Rodriguez M, Egger M: Mortality of patients lost to follow-up in antiretroviral treatment programmes in resource-limited settings: systematic review and meta-analysis. PLOS ONE 2009, 4(6):e5790.

21. Yu JK, Chen SC, Wang KY, Chang CS, Makombe SD, Schouten EJ, Harries AD: True outcomes for patients on antiretroviral therapy who are "lost to follow-up" in Malawi. Bull World Health Organ 2007, 85(7):550-4.

22. Tonwe-Gold B, Ekouevi DK, Bosse CA, Toure S, Koné M, Becquet R, Leroy V, Toro P, Dabis F, El Sadr WM, Abrams EJ: Implementing family-focused HIV care and treatment: the first 2 years' experience of the mother-to-child transmission-plus program in Abidjan, Cote d'Ivoire. Trop Med Int Health 2009, 14(2):204-12.

23. Creek T, Tanuri A, Smith M, Seipone K, Smit M, Legwaila K, Motswere C, Maruping M, Nkoane T, Ntumy R, Bile E, Mine M, Lu L, Tebele G, Mazhani L, Davis MK, Roels TH, Kilmarx PH, Shaffer N: Early diagnosis of human immunodeficiency virus in infants using polymerase chain reaction on dried blood spots in Botswana's national program for prevention of mother-to-child transmission. Pediatr Infect Dis J 2008, 27(1):22-6.

24. Lofgren SM, Morrissey AB, Chevallier CC, Malabeja Al, Edmonds S, Amos B, Sifuna DJ, von Seidlein L, Schimana W, Stevens WS, Bartlett JA, Crump JA: Evaluation of a dried blood spot HIV-1 RNA program for early infant diagnosis and viral load monitoring at rural and remote healthcare facilities. AIDS 2009, 23(18):2459-66

25. Ngo-Giang-Huong N, Khamduang W, Leurent B, Collins I, Nantasen I, Leechanachai P, Sirirungsi W, Limtrakul A, Leusaree T, Comeau AM, Lallemant M, Jourdain G: Early HIV-1 diagnosis using in-house real-time PCR amplification on dried blood spots for infants in remote and resource-limited settings. J Acquir Immune Defic Syndr 2008, 49(5):465-71.

26. Newell ML, Coovadia H, Cortina-Borja M, Rollins N, Gaillard P, Dabis F: Mortality of infected and uninfected infants born to HIV-infected mothers in Africa. a pooled analysis Lancet 2004, 364(9441):1236-43.

27. Kiboneka A, Wangisi J, Nabiryo C, Tembe J, Kusemererwa S, OlupotOlupot P, Joffres M, Anema A, Cooper CL, Montaner JS, Mills EJ: Clinical and immunological outcomes of a national paediatric cohort receiving combination antiretroviral therapy in Uganda. AIDS 2008, 22(18):2493-9.

28. Harries $A D$, Zachariah $R$, Lawn $S D$, Rosen $S$ : Strategies to improve patient retention on antiretroviral therapy in sub-Saharan Africa. Trop Med Int Health 15(Suppl 1):70-5.

\section{Pre-publication history}

The pre-publication history for this paper can be accessed here: http://www.biomedcentral.com/1471-2458/11/519/prepub

doi:10.1186/1471-2458-11-519

Cite this article as: Ekouevi et al:: 12-month mortality and loss-toprogram in antiretroviral-treated children: The leDEA pediatric West African Database to evaluate AIDS (pWADA), 2000-2008. BMC Public Health 2011 11:519.

\section{Submit your next manuscript to BioMed Central and take full advantage of:}

- Convenient online submission

- Thorough peer review

- No space constraints or color figure charges

- Immediate publication on acceptance

- Inclusion in PubMed, CAS, Scopus and Google Scholar

- Research which is freely available for redistribution 